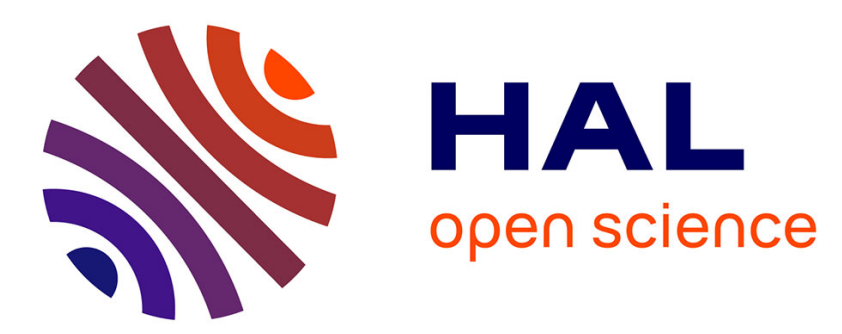

\title{
Etude de la déformée d'une grille de tenségrité pour l'identification de son niveau d'autocontrainte
}

Nicolas Angellier, Jean-François Dubé, Jérôme Quirant, Bernard Crosnier

\section{To cite this version:}

Nicolas Angellier, Jean-François Dubé, Jérôme Quirant, Bernard Crosnier. Etude de la déformée d'une grille de tenségrité pour l'identification de son niveau d'autocontrainte. European Journal of Environmental and Civil Engineering, 2009, 13, pp.1183-1202. 10.1080/19648189.2009.9693185 . hal-00528737

\section{HAL Id: hal-00528737 https://hal.science/hal-00528737}

Submitted on 28 Mar 2012

HAL is a multi-disciplinary open access archive for the deposit and dissemination of scientific research documents, whether they are published or not. The documents may come from teaching and research institutions in France or abroad, or from public or private research centers.
L'archive ouverte pluridisciplinaire HAL, est destinée au dépôt et à la diffusion de documents scientifiques de niveau recherche, publiés ou non, émanant des établissements d'enseignement et de recherche français ou étrangers, des laboratoires publics ou privés. 


\title{
Etude de la déformée d'une grille de tenségrité pour l'identification de son niveau d'autocontrainte
}

\author{
Nicolas Angellier - Jean François Dubé - Jérôme Quirant — \\ Bernard Crosnier
}

LMGC-UMR 5508, Place E. Bataillon, F-34095 Montpellier cedex 5

\{angellier, dube, quirant, crosnier\}@lmgc.univ-montp2.fr

RÉSUMÉ. Ces travaux font le point sur la pertinence de l'utilisation du champ de déplacement des nœuds d'une structure de tenségrité pour identifier son état d'autocontrainte. Il s'agit de déterminer la corrélation entre la précision de la mesure et la précision de l'identification de l'état d'autocontrainte. En utilisant un tachéomètre, on montre que l'identification à partir du champ de déplacement donne d'aussi bons résultats que celle réalisée à partir de la mesure de quelques efforts internes.

ABSTRACT. We study the possibility to use the measurement of the displacement fields of the nodes of a tensegrity structure under static loading to obtain a new method for the identification of its self-stress state. We try to determinate the correlation between the precision of this identification and the precision of the measure. With a tacheometer we obtain a precision of identification as good as the standard method using efforts measurements.

MOTS-CLÉS : tenségrité, tachéomètre, autocontrainte, mesure de champ, analyse inverse.

KEYWORDS: tensegrity, tacheometer, self-stress, field measurement, inverse analysis.

Revue. Volume $\mathrm{X}-\mathrm{n}^{\circ} \mathrm{x} /$ année, pages 1 à $\mathrm{X}$ 


\section{Introduction}

Depuis de nombreuses années, l'équipe Conception en Structures du Laboratoire de Mécanique et Génie Civil de Montpellier mène des études sur les structures de tenségrité. A la suite du projet «Tensarch ${ }^{\circledR}$ » (Motro, 2002), elle dispose de structures grandeur nature qui lui permettent de mettre en application, développer et confronter les résultats théoriques et numériques mis en œuvre jusqu'à présent. Par exemple, des méthodes originales ont été mises au point pour le contrôle de l'autocontrainte (identification de l'état interne, détermination par calcul numérique d'une variation des longueurs des éléments à corriger pour atteindre un état cible) et le contrôle actif du comportement (maintien d'une géométrie, atténuation des vibrations) (Averseng et al., 2004). Dernièrement, la mise en place de moyens de mesure directe des efforts dans les éléments a permis d'affiner le contrôle de l'autocontrainte en montrant que les mesures indirectes utilisées auparavant présentaient certaines faiblesses (Angellier et al., 2006). Pour poursuivre l'étude de ces grilles, l'équipe s'est dotée d'un nouvel outil performant : un tachéomètre qui mesure la position de tous les nœuds de la structure. Il nous permet d'étudier les champs de déplacements des nœuds de la structure soumise à un chargement statique. Une étude numérique est menée pour étudier la sensibilité de ces champs de déplacements au niveau d'autocontrainte. Une étude expérimentale lui succède pour estimer la précision d'identification du niveau en utilisant le dispositif de mesure tachéomètrique.

\section{Identification de l'état interne}

\subsection{La minigrille}

Il s'agit d'une grille plane à double nappe de câbles dont le motif de base reprend le principe de l'écarteur : 2 à 2 les barres forment des $\mathrm{V}$ perpendiculaires et opposés séparés par un tirant vertical dont on peut faire varier la longueur. Elle est constituée de 81 éléments reliés par des nœuds : 24 barres (tubes) en compression et des éléments en tension, à savoir 36 câbles ainsi que 9 tirants verticaux et 12 tirants périphériques; les tirants sont les éléments actifs de la structure (figure 1). Cette grille a été conçue de manière à répondre à la définition de structure de tenségrité : « système dans un état d'auto équilibre stable (ici autocontrainte), qui comprend un ensemble discontinu de composants comprimés (les barres) à l'intérieur d'un continuum de composants tendus (les câbles et les tirants)» (Motro, 2003). 


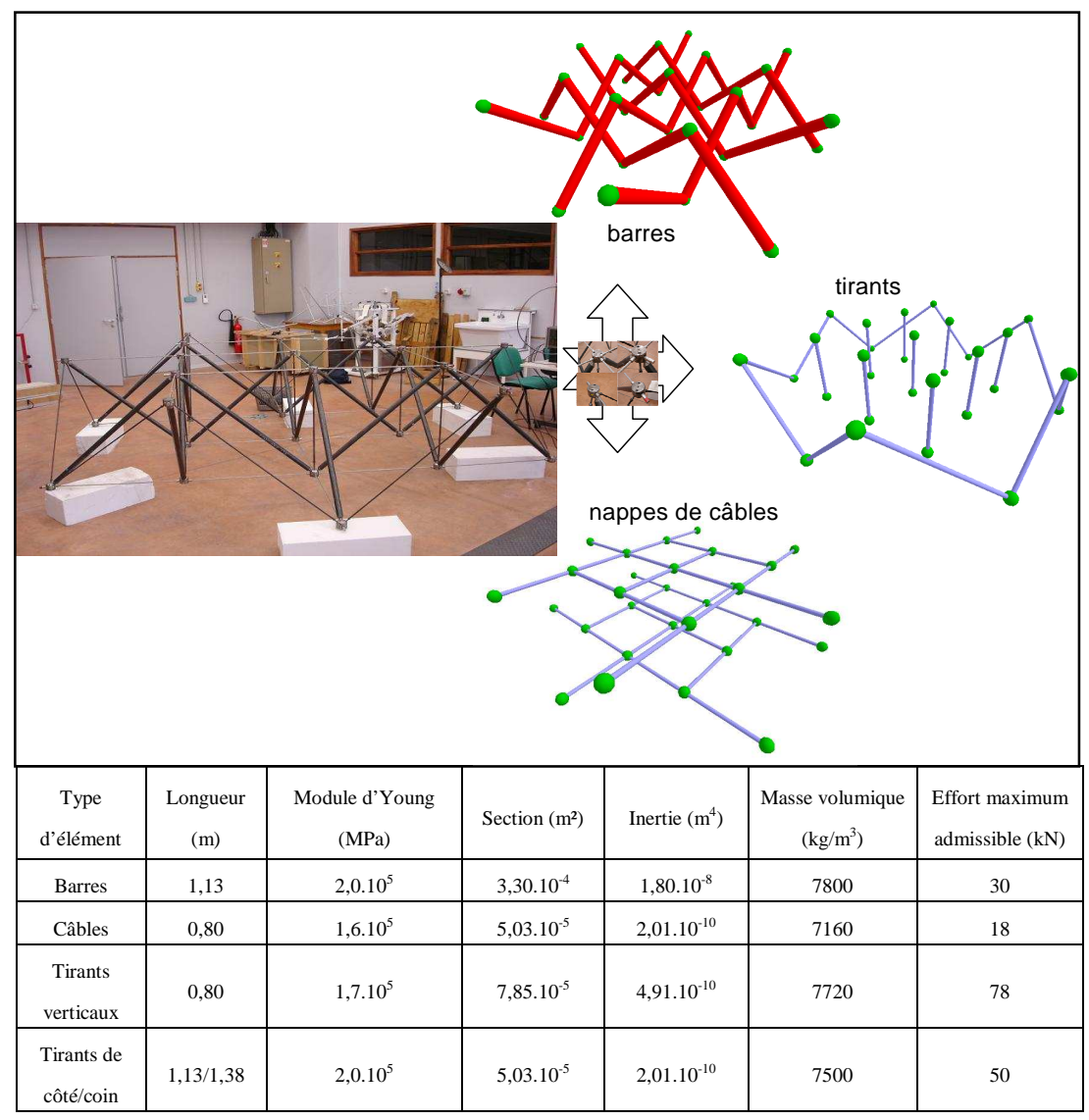

Figure 1. La minigrille, caractéristiques des éléments, modélisation 


\subsection{Etat d'autocontrainte}

L'équilibre d'un système est obtenu quand tous ses nœuds sont en équilibre. L'équilibre statique d'un nœud $i$ s'écrit comme suit :

$$
\sum_{j \neq i} T_{i j}+F_{i}=0
$$

$T_{i j}$ représente l'effort interne de l'élément reliant le nœud $j$ au nœud $i$, et $F_{i}$ l'effort extérieur appliqué au nœud $i$. Les efforts internes sont fonctions de l'allongement de chaque élément. On note $x_{i}, y_{i}, z_{i}$ les coordonnées spatiales du nœud $i$, et $l_{i j}^{0}$ la longueur de l'élément reliant les nœuds $i$ et $j$ dans la configuration géométrique de référence. La projection de l'équation [1] sur les 3 axes donne :

$$
\begin{aligned}
& \sum_{j \neq i} T_{i j} \frac{x_{j}-x_{i}}{l_{i j}^{0}}+F_{i}{ }^{x}=0 \\
& \sum_{j \neq i} T_{i j} \frac{y_{j}-y_{i}}{l_{i j}^{0}}+F_{i}{ }^{y}=0 \\
& \sum_{j \neq i} T_{i j} \frac{z_{j}-z_{i}}{l_{i j}^{0}}+F_{i}{ }^{z}=0
\end{aligned}
$$

Cette équation d'équilibre peut être simplifiée en introduisant la densité de force $q_{i j}$ de chaque élément $(i, j)$ :

$$
q_{i j}=\frac{T_{i j}}{l_{i j}^{0}}
$$

La relation [2] pour un nœud devient alors :

$$
\begin{aligned}
& \sum_{j \neq i} q_{i j}\left(x_{j}-x_{i}\right)+F_{i}{ }^{x}=0 \\
& \sum_{j \neq i} q_{i j}\left(y_{j}-y_{i}\right)+F_{i}{ }^{y}=0 \\
& \sum_{j \neq i} q_{i j}\left(z_{j}-z_{i}\right)+F_{i}{ }^{z}=0
\end{aligned}
$$

Le système d'équations obtenu en appliquant les équations [4] à tous les nœuds de la structure est la forme globale matricielle suivante :

$$
A q=f
$$


Avec $\boldsymbol{A}$ la matrice d'équilibre de la structure (de dimension $b \times 3 n$ ), $\boldsymbol{q}$ le vecteur des densités de forces des $b$ éléments, et $f$ le vecteur du chargement extérieur agissant sur les $n$ nœuds.

L'autocontrainte est l'ensemble des forces internes présent à l'état initial en l'absence de chargement extérieur. Cet état d'équilibre correspond à un champ de densité de forces $\boldsymbol{q}_{0}$ satisfaisant :

$$
A q_{0}=0 \Leftrightarrow q_{0} \quad k e r A
$$

L'état d'autocontrainte peut être exprimé sur la base du sous-espace kerA. Cette base, notée $\boldsymbol{S}$, est composée de plusieurs états d'autocontrainte fondamentaux. Un état fondamental peut être de 2 natures : s'il sollicite tous les composants d'un système il est total, dans le cas contraire il est partiel. La base $S$ doit permettre de construire un état d'autocontrainte total.

La base d'autocontrainte $S$ ne peut être utilisée directement car la matrice d'équilibre $\boldsymbol{A}$ ne tient pas compte du comportement unilatéral des éléments. L'état d'autocontrainte qui y satisfait est appelé conforme. Il peut être créé à partir d'une base d'autocontrainte constituée d'états conformes (Sanchez, 2005) ou directement par combinaison linéaire de la base $S$ (Quirant et al., 2003) :

$$
q_{0}=S \alpha
$$

Les composantes du vecteur $\boldsymbol{\alpha}$ sont choisies pour satisfaire la conformité du comportement mécanique des éléments.

\subsection{Etat d'autocontrainte de la minigrille}

Le logiciel Tenségrité 2000 (Quirant, 2000) nous permet de déterminer les états d'autocontrainte de base d'un système de tenségrité. Pour ce faire, 2 facteurs rentrent en jeu :

- les conditions d'appuis : ici 3 appuis bloqués et répartis non symétriquement,

- les données géométriques : position des nœuds et connectivité des éléments.

Ainsi, la minigrille ne possède que 2 états d'autocontrainte de base partiels : un état local $E A 1$ et un état diffus $E A 2$. La figure 2 montre bien que pour l'état EA1, les éléments périphériques n'interviennent pas dans l'équilibre (couleur blanche), alors que pour l'état EA2, cela ne concerne que quelques câbles. En multipliant ces états de base avec les longueurs libres $l_{i}$ des éléments, on obtient les vecteurs d'effort interne $[T i]=E A i \times l_{i}$. Les 2 vecteurs [T1] et [T2] ainsi calculés peuvent être combinés à l'aide de 2 coefficients pondérateurs indépendants $a$ et $b$. La combinaison linéaire $a_{\times}[T 1]+b_{\times}[T 2]$, permet de construire un état d'autocontrainte total qui assure la stabilité de la structure et les états limites de service que nous imposons. 


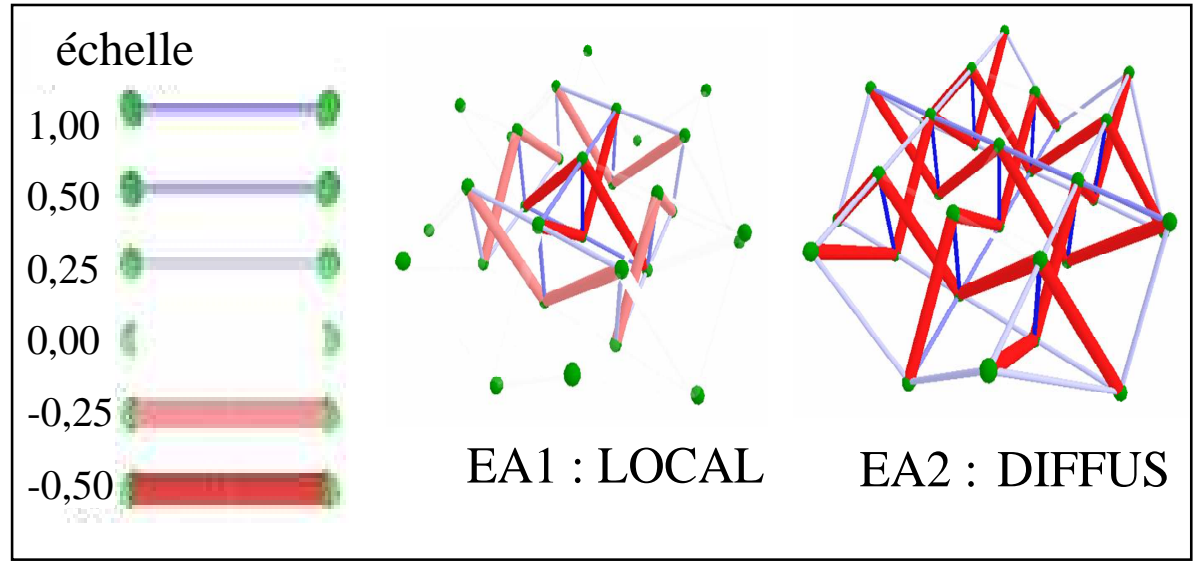

Figure 2. Les états d'autocontrainte de base de la minigrille

C'est pour un rapport 0,5 entre les 2 coefficients $a$ et $b$ de chaque vecteur d'effort que l'on obtient une répartition des forces dans les éléments la plus homogène possible, notamment pour les tensions des câbles ; éléments qui pilotent la rigidité de la structure. Ce rapport étant fixé, c'est le paramètre $a$ qui est utilisé comme indicateur du niveau d'autocontrainte de cet état cible privilégié pour la structure étudiée. On fait varier ce niveau global pour atteindre des valeurs réalistes des efforts dans les éléments (état réaliste) qui permettent d'avoir une structure suffisamment rigide sans atteindre le flambement des barres (qui intervient pour $a$ supérieur à 6600) : c'est par exemple le cas pour $a=2000$ (figure 3).

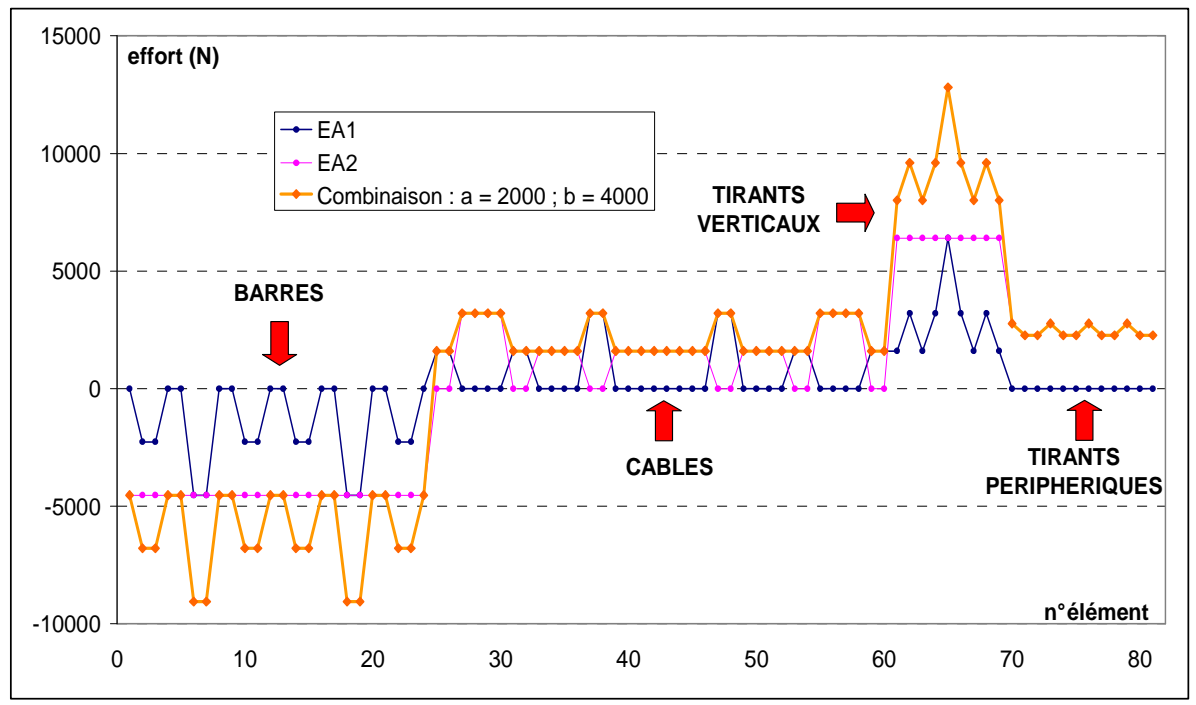

Figure 3. Combinaison des vecteurs d'effort de la minigrille pour un état réaliste 
Pour identifier l'état interne $\alpha_{i d}$ de la grille (c'est-à-dire ici déterminer les coefficients $a^{\prime}$ et $b^{\prime}$ pour la structure) nous utilisons une méthode qui consiste en la mesure des efforts d'un nombre limité d'éléments $\left\{T_{m e s}\right\}$. Ici nous mesurons 4 barres centrales, 2 tirants verticaux (un de coin et un de côté) et 1 tirant périphérique, à l'aide de montages de jauges dédiés (figure 4). Nous calons sur le résultat de ces mesures un état généré par la base d'autocontrainte (formée par les vecteurs d'effort [Ti] déterminés précédemment). Pour cela, nous utilisons la méthode de l'inverse généralisée ou pseudo inverse pour minimiser la différence entre l'état généré et l'état mesuré (Averseng, 2004). Nous avons montré auparavant que l'influence d'une géométrie réelle sur la détermination de ces états est faible (Angellier, 2007) : elle ne remet pas en cause la validité de la méthode et le comportement mécanique de la minigrille en est très peu affecté. L'utilisation de la géométrie théorique parfaite suffit donc pour l'identification de l'état d'autocontrainte de la structure. Cette identification initiale de l'état d'autocontrainte de la grille, établi lors de sa mise en tension et qui nous sert de référence, donne le coefficient $a^{\prime}=1720$ et le coefficient $b^{\prime}=3200$, soit un rapport $a^{\prime} / b^{\prime}=0,45$, proche du rapport ciblé. L'imprécision maximum sur les efforts est de $14 \%$ pour le tirant vertical de coin (figure 4). Cela conduit à une erreur maximale sur le coefficient $a$ ' identifié inférieure à 250 (soit inférieure à $15 \%$ ). Il est possible d'atteindre une précision inférieure à $5 \%$ sur l'état d'autocontrainte. Toutefois, pour la présente étude, un écart de $14 \%$ est amplement suffisant. 


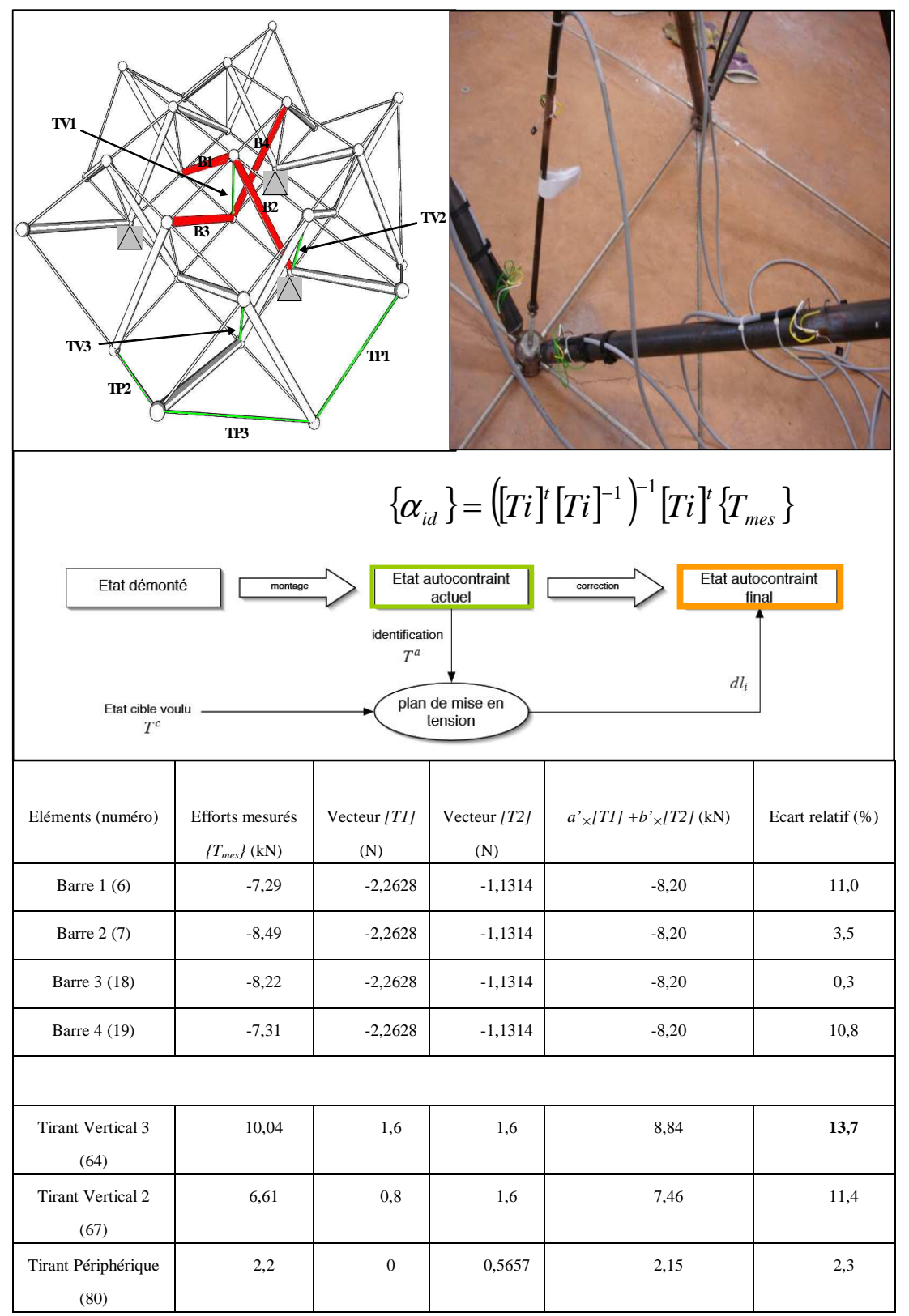

Figure 4. Combinaison des vecteurs de tension de la minigrille : identification expérimentale par mesure d'efforts 


\section{Simulation du champ de déplacements des nœuds de la structure chargée}

\subsection{Déformée de la minigrille sous chargement statique}

Pour calculer un treillis, on pose généralement l'hypothèse d'un comportement de type BARRE pour la modélisation de tous les éléments d'une structure de tenségrité. Dans notre cas, cette hypothèse est remise en cause par une étude antérieure (Angellier et al., 2006) où nous avons détecté de la flexion dans les barres en état d'autocontrainte. Nous avons montré en statique et dynamique que le comportement des éléments comprimés et des tirants était de type POUTRE. Les câbles sont modélisés par des éléments BARRE.

La rigidité de la grille étant élevée dès la mise en autocontrainte nous devons appliquer des efforts suffisamment importants pour obtenir une déformée mesurable. Des tests numériques rapides montrent qu'un chargement ponctuel doit être excessivement élevé : par exemple, avec des conditions d'appuis favorables, la simulation d'un chargement de presque $10 \mathrm{kN}$ appliqué au nœud central de la nappe supérieure donne des déplacements maximums de l'ordre de 0,2 $\mathrm{mm}$ seulement. Cela conduit à des difficultés techniques de mise en place mais surtout à des risques de perte d'autocontrainte (pertes de tension dans les câbles, barres défaites) qui mettraient en défaut la conformité de la structure. Pour ces raisons, nous optons pour l'application successive de charges aux 6 nœuds périphériques de la nappe inférieure qui permet d'atteindre un chargement total efficient (figure 5).

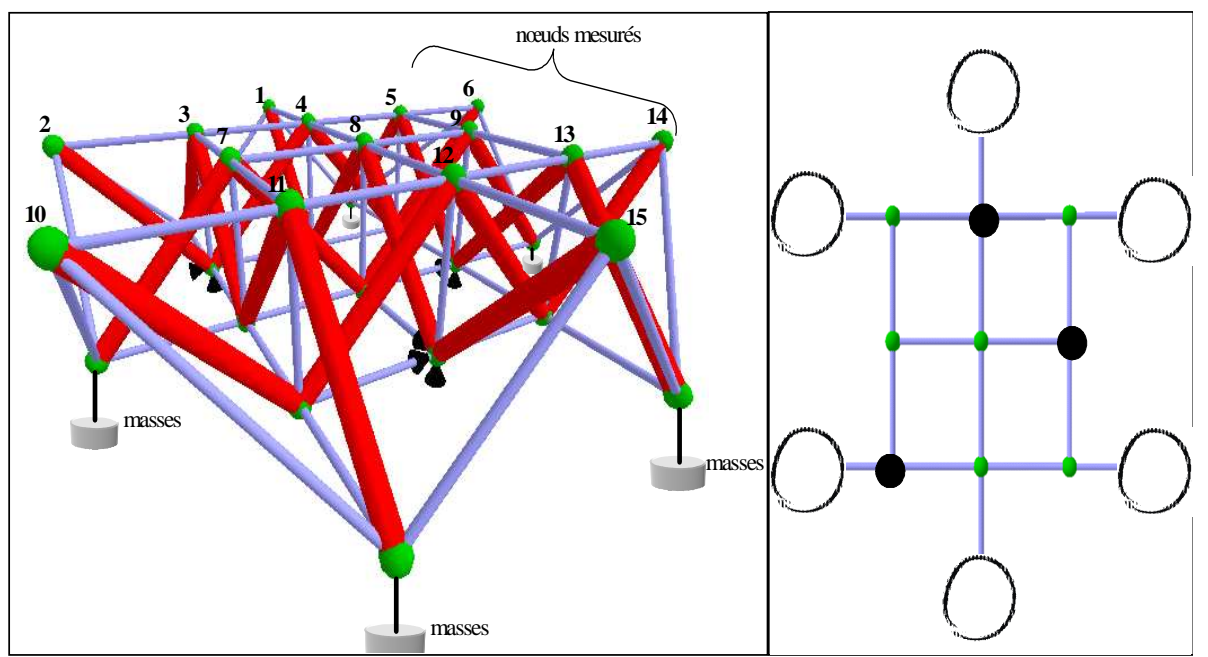

Figure 5. Stratégie de chargement de la nappe inférieure de la minigrille et de mesure des champs de déplacements induits sur les nœuds de la nappe supérieure 
Nous présentons les résultats de la simulation avec le logiciel éléments finis cast3m (Verpeaux et al., 1988) avec un chargement allant jusqu'à $315 \mathrm{~N}$ par nœud chargé (soit un total de $1,89 \mathrm{kN}$ ). L'état d'autocontrainte est fixé avec un rapport des coefficients de base $a$ et $b$ de 0,5 et le coefficient $a$ est fixé à 2000 (figure 3). Nous nous intéressons aux déplacements verticaux et horizontaux des 15 nœuds de la nappe supérieure. Les déplacements des nœuds sont linéaires en fonction du niveau de charge (figure 6).
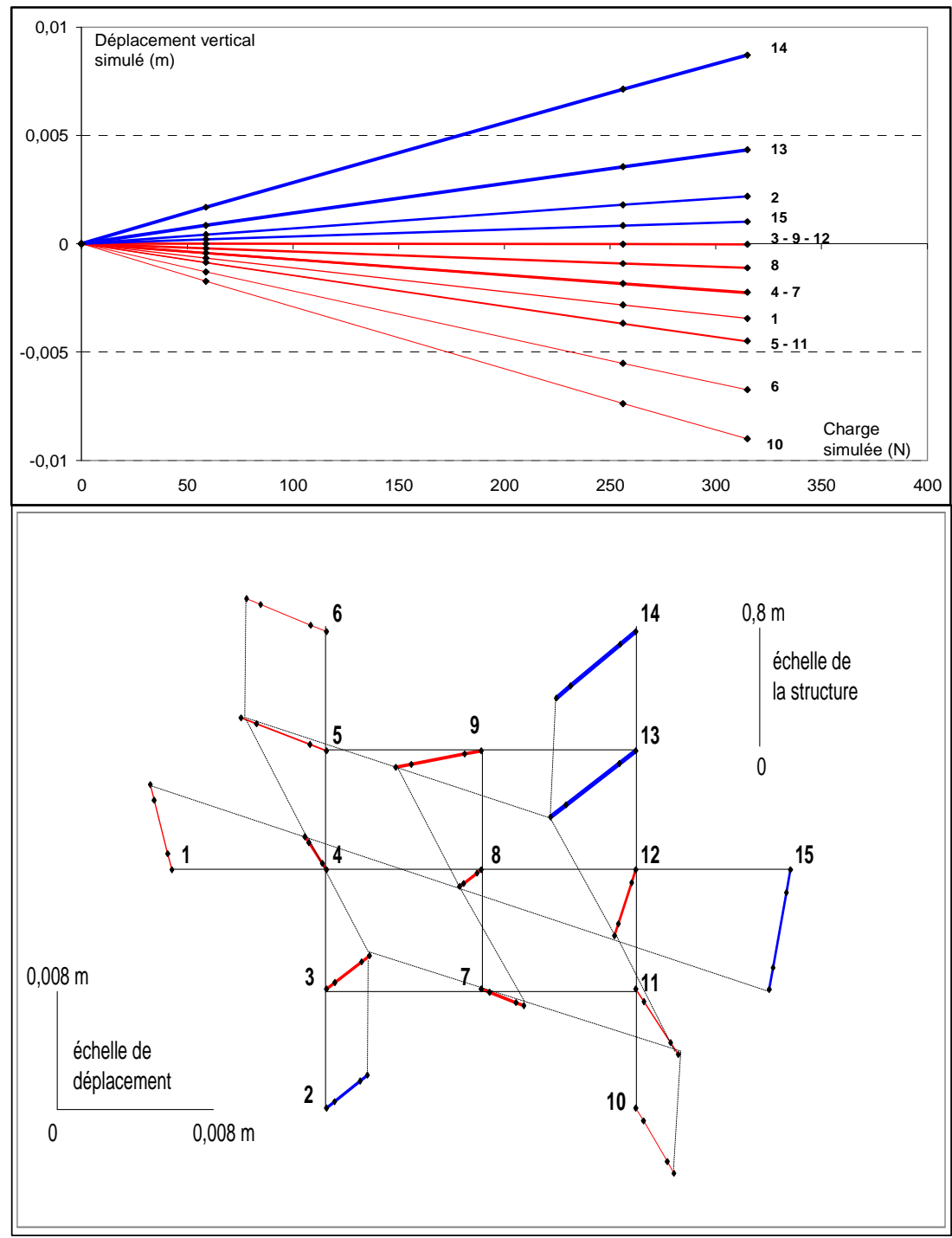

Figure 6. Simulation des champs de déplacements verticaux et horizontaux pour un état d'autocontrainte réaliste 
On peut noter que les déplacements verticaux de la majorité des nœuds suivent le sens du chargement en descendant sauf pour 4 nœuds $(2,13,14$ et 15$)$ qui montent. Ces nœuds sont situés derrière les 3 nœuds reliés par des tirants verticaux aux nœuds des appuis (3, 9 et 12) dont les déplacements sont quasiment nuls. Enfin, les 2 nœuds pour lesquels les déplacements verticaux sont maximums (proches de $1 \mathrm{~cm}$ ) sont des nœuds périphériques $(10,14)$.

Pour les déplacements horizontaux, la figure 6 montre la nappe de nœuds supérieure vue du dessus, ce qui permet d'observer sa déformée sous chargement par le mouvement d'ensemble de ses nœuds. On distingue une rotation et un double cisaillement de part et d'autre des lignes de nœuds passant par le nœud central (8) dont le déplacement est minimal. Les déplacements horizontaux maximums qui dépassent ici $5 \mathrm{~mm}$ sont obtenus pour les nœuds de la périphérie (en particulier le nœud 15).

\subsection{Sensibilité de la déformée au niveau d'autocontrainte}

Nous approfondissons cette étude numérique en cherchant à déterminer la sensibilité des champs de déplacements des nœuds par rapport à la variation du niveau d'autocontrainte autour d'un état de conception. Nous faisons donc varier le coefficient $a$ tout en conservant le rapport $a / b$ fixe.

La figure 7 montre les résultats obtenus pour un chargement simulé de $256 \mathrm{~N}$ par nœud chargé, en explorant la gamme des niveaux d'autocontrainte possibles: les valeurs du coefficient $a$ vont de 1000 (état peu contraint) à 6000 (état approchant la limite de flambement des barres). De manière générale, nous observons une diminution des déplacements en valeur absolue avec l'augmentation de l'autocontrainte.

Plus précisément, pour a variant dans l'intervalle 1500-2500 autour de l'état d'autocontrainte de conception, les nœuds 10 et 14 ont les déplacements verticaux les plus sensibles à la variation du niveau d'autocontrainte. Pour les déplacements horizontaux, le nœud 15 est le plus sensible. Ces nœuds sont aussi les nœuds dont les déplacements sont les plus importants. Pour ces 3 nœuds, les déplacements diffèrent de près de $1,5 \mathrm{~mm}$ entre les 2 états d'autocontrainte. Pour le même intervalle d'autocontrainte, nous estimons la différence moyenne des déplacements sur les 15 nœuds : elle est de $0,57 \mathrm{~mm}$ pour le déplacement vertical et $0,76 \mathrm{~mm}$ pour le déplacement horizontal. Le déplacement horizontal est donc légèrement plus sensible à la variation d'autocontrainte. Il est à noter que les directions des déplacements horizontaux ne varient pas avec le niveau d'autocontrainte. En fait, elles ne varient pas non plus avec le rapport $a / b$ : elles semblent seulement caractéristiques des conditions d'appuis de la grille et de l'application des charges. 

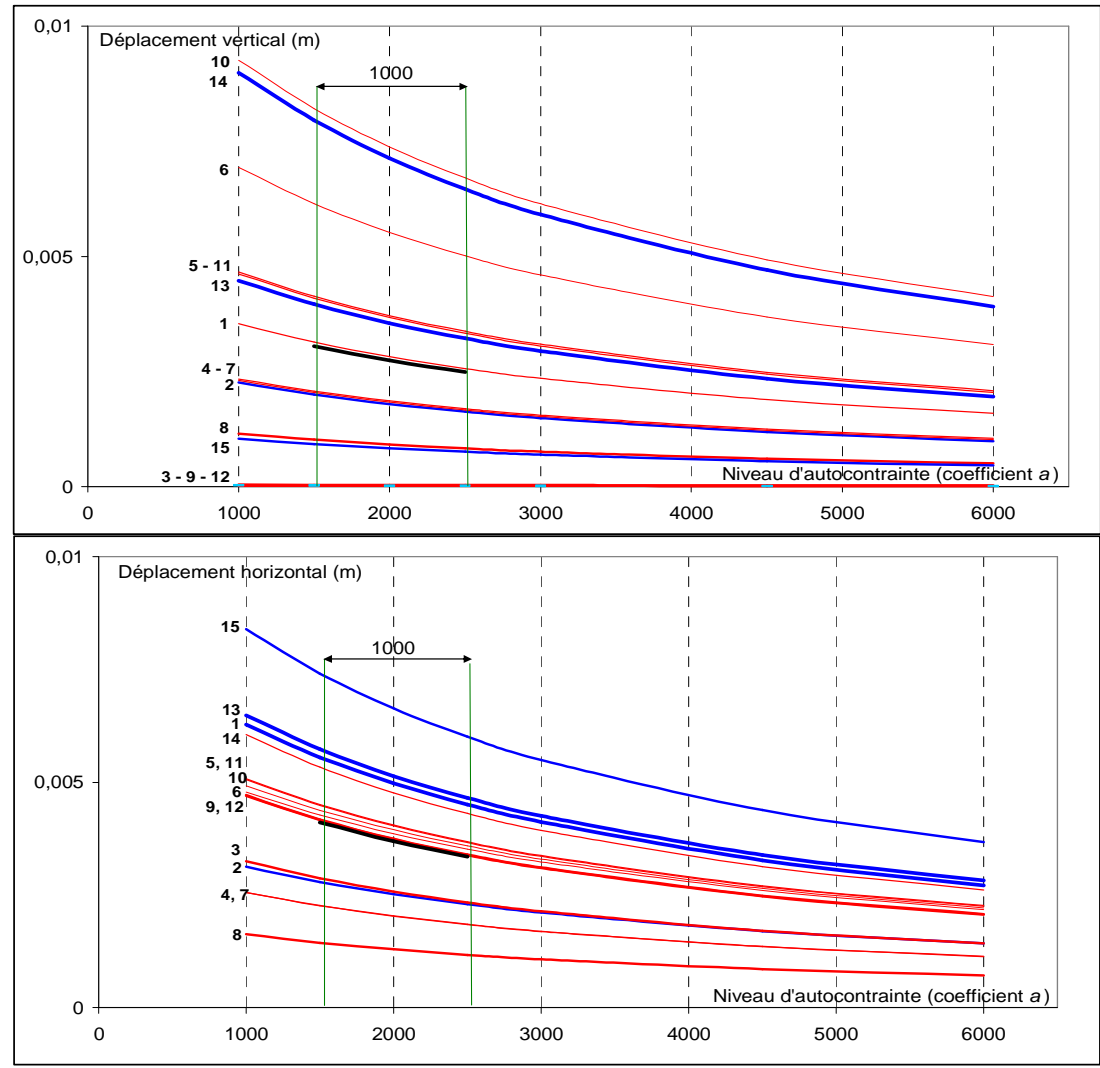

Figure 7. Variation des champs de déplacements des næuds en fonction du niveau d'autocontrainte

Pour l'intervalle d'autocontrainte fixé, en établissant les différences moyennes des déplacements obtenus pour d'autres chargements, on constate que leur évolution en fonction de la charge appliquée peut être linéarisée (figure 8). La valeur des pentes des droites nous permet de prédire ces différences moyennes des déplacements pour un chargement quelconque :

$$
\begin{aligned}
& D_{V-1000}\left(10^{-4} \mathrm{~m}\right)=0,022 \times C_{6}(N) \\
& D_{H-1000}\left(10^{-4} \mathrm{~m}\right)=0,030 \times C_{6}(N)
\end{aligned}
$$

Avec $\mathrm{D}_{\mathrm{V}-1000}$ et $D_{H-1000}$ les différences moyennes verticale et horizontale des déplacements pour un intervalle de $a$ d'étendue $1000, C_{6}$ le chargement appliqué aux 6 nœuds périphériques de la nappe inférieure. 
Ainsi, en connaissant la précision du dispositif expérimental de mesure des déplacements, il nous est possible de donner le chargement expérimental minimal permettant la mesure d'un champ de déplacements des nœuds qui permet de distinguer des niveaux d'autocontraintes suffisamment proches. Par exemple, nous pouvons chercher à distinguer 2 états d'autocontrainte réalistes dont la différence des coefficients $a$ vaut 200 (soit une précision d'environ $10 \%$ pour des états proches de l'état de niveau $a=2000$ ). Si l'on veut se contenter d'un chargement de $59 \mathrm{~N}$ sur chacun des 6 nœuds périphériques de la nappe inférieure, les équations [8] et [9] nous donnent la précision minimale du dispositif expérimental de mesure des déplacements : il est de $0,022 \times 59 \times(200 / 1000)=0,026 \mathrm{~mm}$ pour le déplacement vertical et $0,030 \times 59 \times(200 / 1000)=0,035 \mathrm{~mm}$ pour le déplacement horizontal. En ciblant les nœuds les plus sensibles, cette précision minimale peut être revue à la hausse. Le paragraphe suivant présente les résultats expérimentaux et la comparaison avec les simulations. Nous utilisons cette démarche pour déterminer la précision de l'identification du niveau d'autocontrainte de la minigrille avec notre dispositif expérimental.

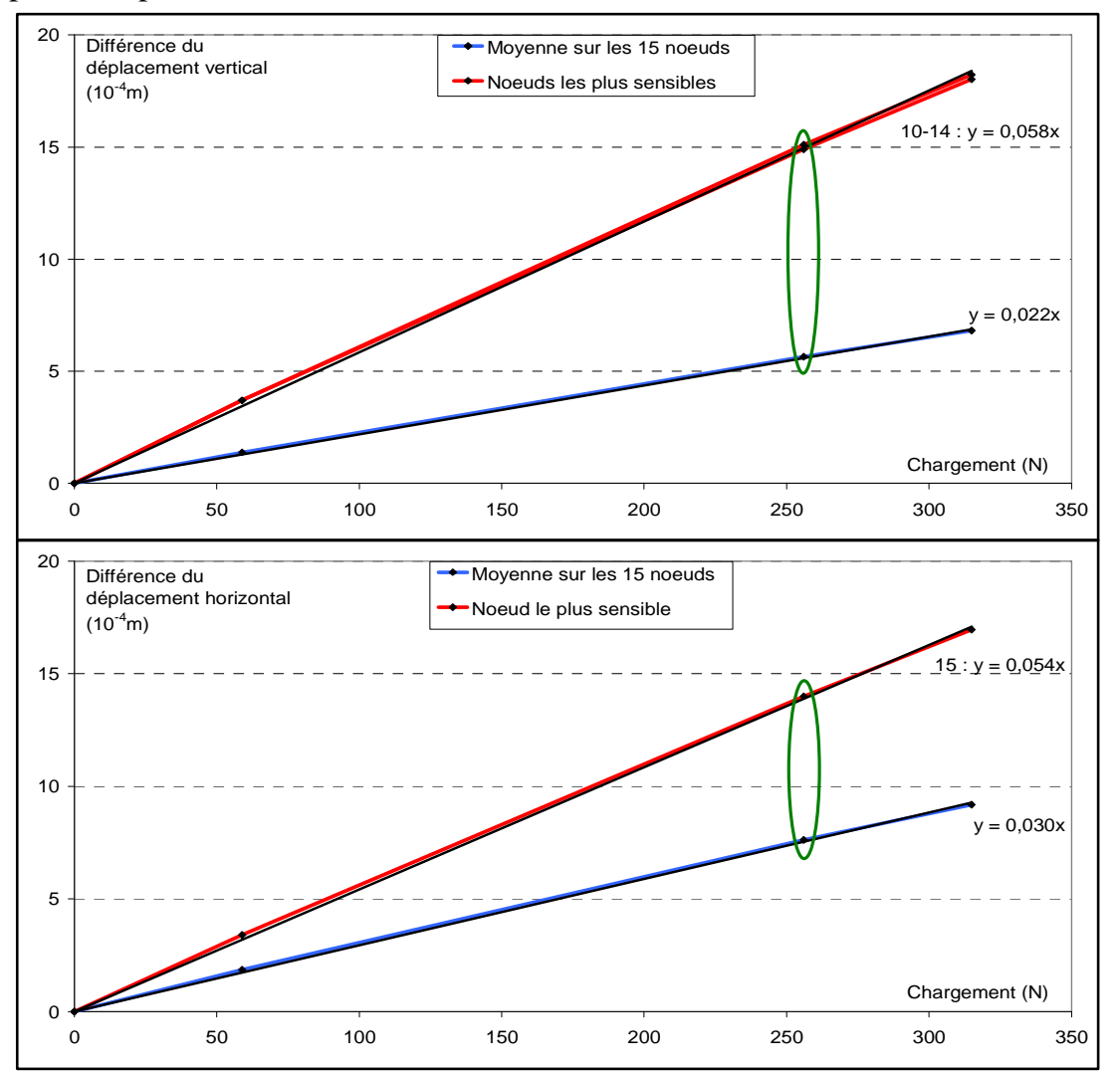

Figure 8. Différences moyennes des déplacements pour un intervalle de a égal à 1000 en fonction du chargement 


\section{Mesure du champ de déplacements des nœuds de la structure chargée}

\subsection{Mesure de la déformée de la minigrille sous chargement statique}

Pour qualifier une méthode d'identification de l'état d'autocontrainte d'une structure de tenségrité en service, nous cherchons à cartographier la déformée expérimentale de la structure entière, par nappe complète. Pour cela, nous disposons d'un tachéomètre LEICA TDA5005, associé à des cibles réflecteurs coin cube creux CCR 1,5, qui affiche une précision de 0,2 mm. Des supports spécifiques ont été usinés et placés sur les nœuds pour pouvoir fixer et centrer la cible du tachéomètre (figure 9): la répétition des mesures de position des nœuds de la minigrille en autocontrainte non chargée montre qu'ils offrent effectivement une précision moyenne verticale de $0,2 \mathrm{~mm}$. La précision n'est cependant que de $1 \mathrm{~mm}$ pour le positionnement horizontal de la cible.

Ainsi, pour un chargement de $315 \mathrm{~N}$, la précision initiale affichée du dispositif tachéomètre-cibles offre la possibilité d'identifier des niveaux d'autocontrainte réalistes proches du niveau 2000 à plus ou moins 300 pour le déplacement vertical (équation [8] : $0,022 \times 315 \times(\Delta a / 1000)=0,2 \mathrm{~mm})$ et à plus ou moins 210 pour le déplacement horizontal (équation [9] : $0,030 \times 315 \times(\Delta a / 1000)=0,2 \mathrm{~mm})$. Cependant, pour ce dernier calcul, la précision de positionnement de la cible sur les supports spécifiques ne permet pas d'espérer pouvoir identifier les niveaux d'autocontrainte avec une telle précision (on trouve en fait un $\Delta a$ de 1000). C'est donc bien avec la coordonnée verticale qu'il faut s'attendre à avoir les meilleurs résultats, de l'ordre de $15 \%$, pour l'identification du niveau d'autocontrainte.

Nous allons confronter la mesure du champ de déplacements des nœuds de la nappe supérieure au champ donné par la simulation dans le cas d'une série de chargements pour un état d'autocontrainte proche d'un état réaliste (figure 9). 


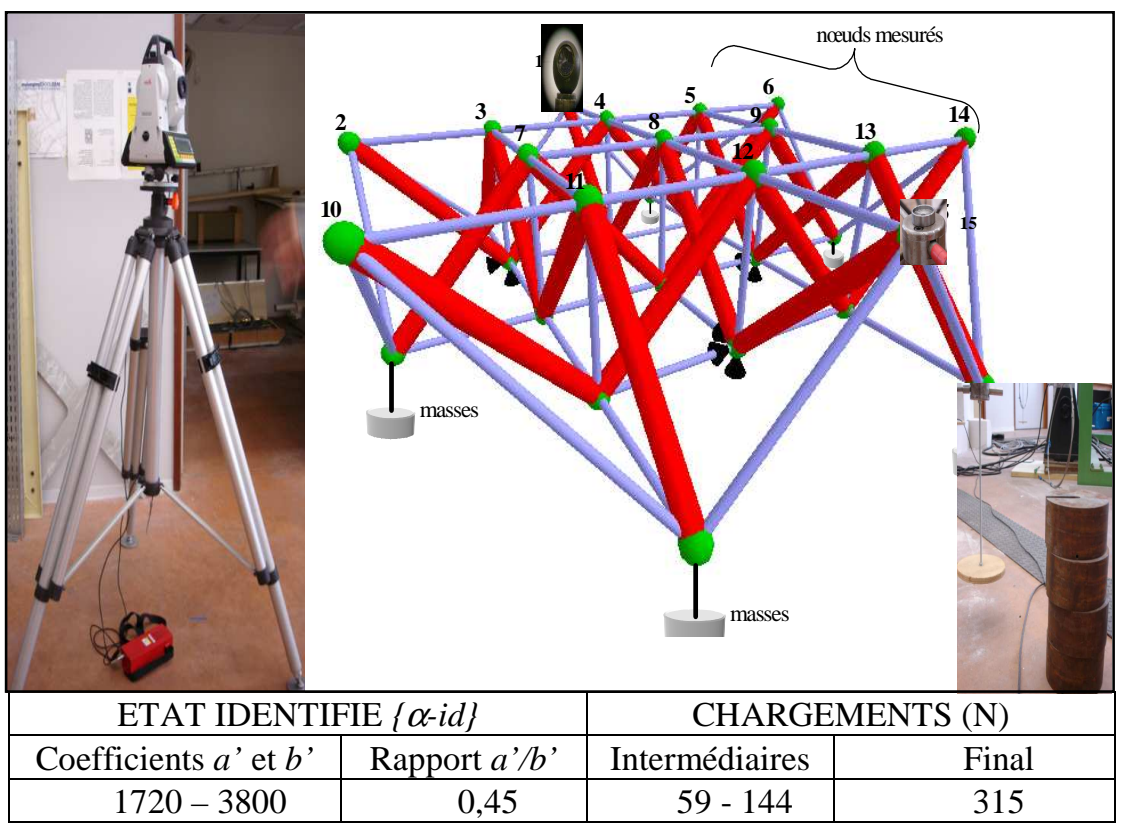

Figure 9. Le tachéomètre, un réflecteur, ses logements, application des charges

Qualitativement, nous retrouvons un champ de déplacements similaire à la simulation (figure 10) : verticalement, les nœuds 2, 13, 14 et 15 montent, les nœuds 3,9 et 12 à l'aplomb des appuis ont logiquement des déplacements quasiment nuls et les nœuds 10 et 14 ont les déplacements maximums. De plus, les pas de charges permettent de visualiser l'évolution linéaire des déplacements. Horizontalement, on peut noter que les directions de déplacements sont en accord avec la simulation.

D'un point de vue quantitatif, les déplacements simulés sous-estiment légèrement les déplacements expérimentaux. Ces écarts peuvent être classiquement imputés à une modélisation purement théorique ne tenant pas compte des jeux et des imperfections des liaisons. Mais nous pouvons aussi y ajouter les fluctuations des efforts dans les éléments constatées au travers des mesures et qui témoignent d'un écart entre l'état d'autocontrainte réel et celui considéré parfait de la simulation. 
16 Revue. Volume $\mathrm{X}-\mathrm{n}^{\circ} \mathrm{x} /$ année

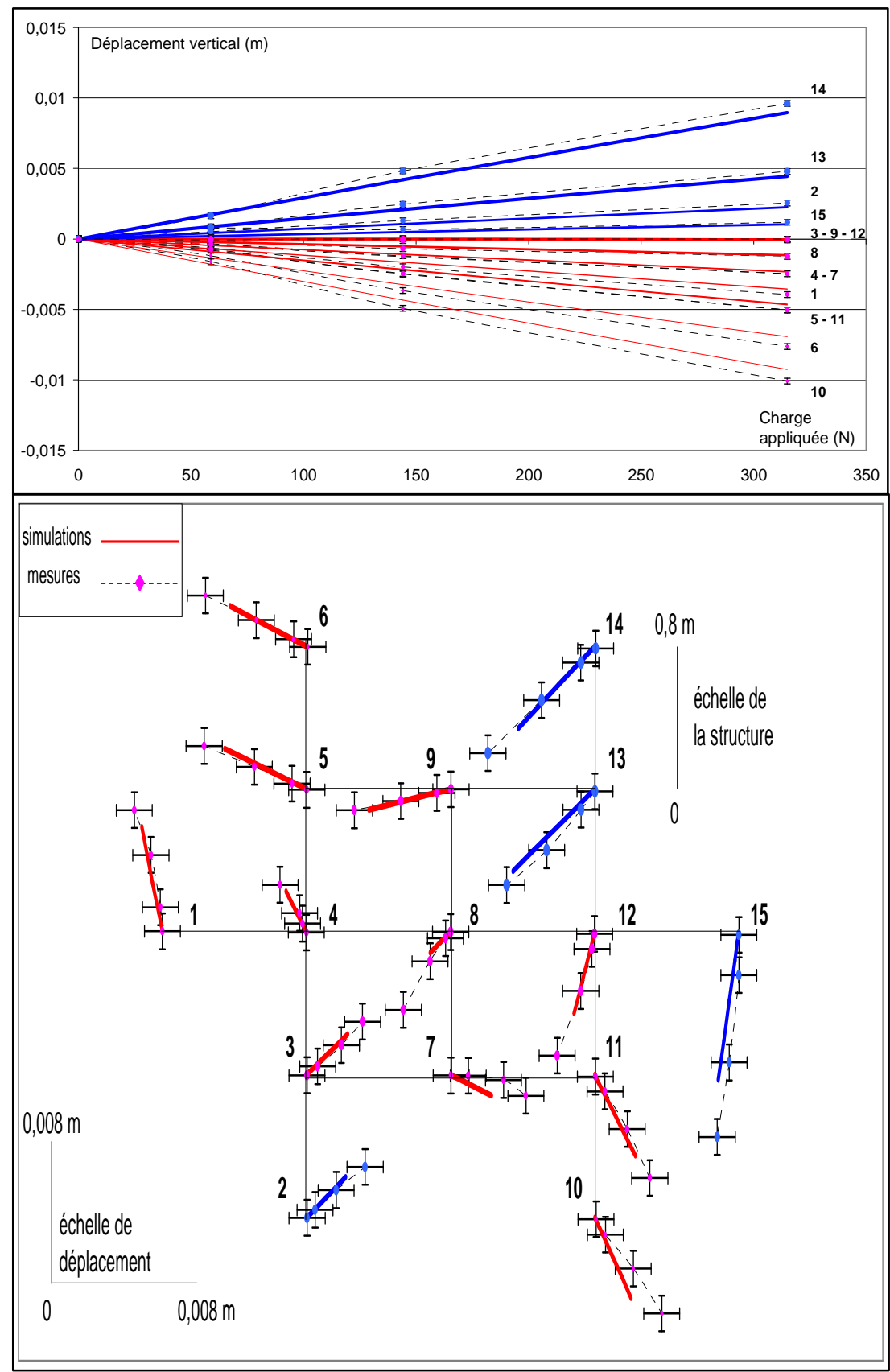

Figure 10. Mesure des champs de déplacements des 15 næuds de la nappe supérieure - Comparaison avec les simulations 
La figure 11 présente la comparaison entre l'évolution des efforts simulés et la mesure des éléments équipés de montages de jauges lors du chargement. L'identification initiale de l'état d'autocontrainte de la grille effectuée grâce à ces mesures, qui nous sert de référence pour la simulation, souffre dès le départ d'une imprécision non négligeable sur ces efforts (tableau de la figure 4). Si cela se traduit par des points de départ différents entre les efforts mesurés et simulés, nous constatons néanmoins des évolutions d'efforts semblables en fonction de la charge appliquée.

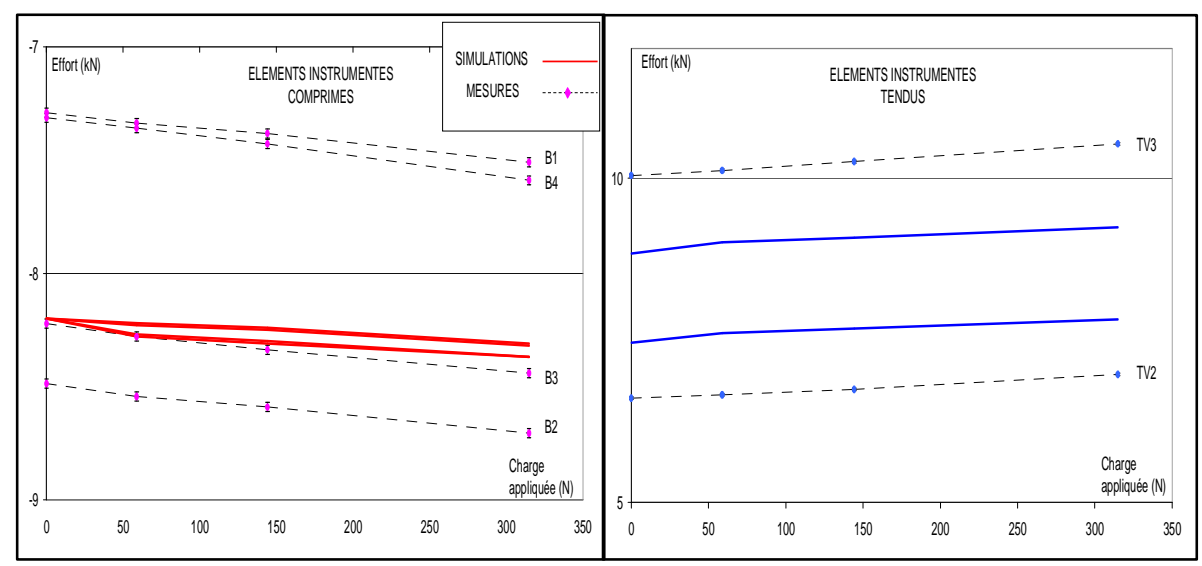

Figure 11. Mesure des efforts des 6 éléments instrumentés - Comparaison avec les simulations

\subsection{Précision de la mesure de champ de déplacement et identification du niveau}

\section{d'autocontrainte}

Les résultats obtenus sont synthétisés tableau 1 en donnant les écarts moyens sur les 15 nœuds entre les mesures expérimentales et les simulations numériques. On retrouve le déplacement vertical, le déplacement horizontal, la direction de ce dernier (sous forme d'angle) ainsi que la variation des efforts lors du chargement. Nous avons associé à ces résultats le calcul des intervalles $\Delta a$ identifiables en considérant que l'écart constaté entre la mesure et la simulation pouvait s'apparenter à la précision du dispositif expérimental. 


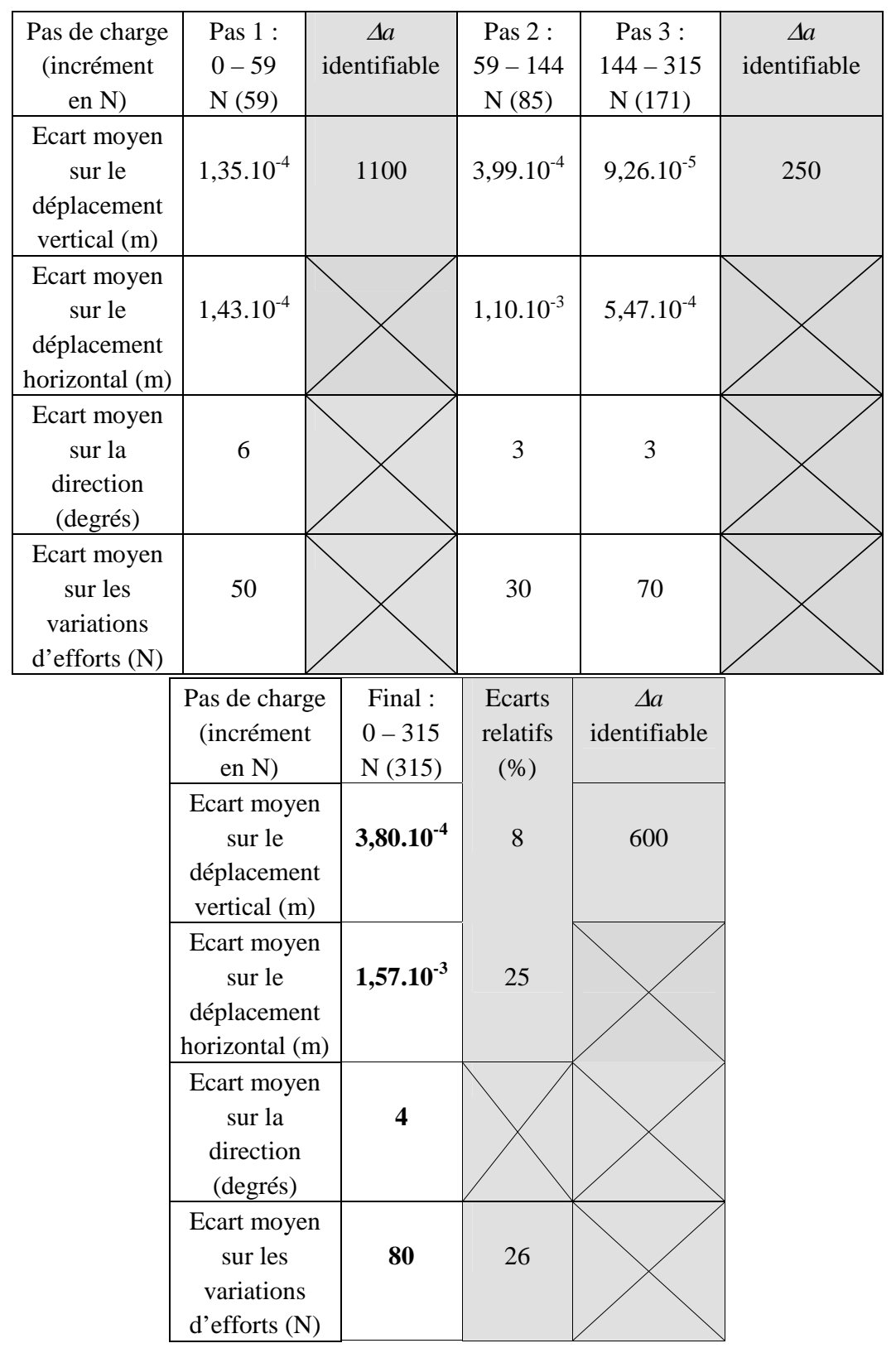

Tableau 1. Ecarts constatés avec la simulation sur les mesures de déplacements et variations d'efforts lors d'un chargement statique - Détermination de l'intervalle de niveau d'autocontrainte identifiable 
On observe que les écarts sur les variations d'effort dans les éléments instrumentés ne sont pas négligeables puisqu'ils dépassent les 25\%, mais nous sommes proches de la limite de sensibilité estimée à $40 \mathrm{~N}$ pour les barres et les tirants lors de l'étalonnage des montages de jauges. On voit aussi que les écarts sur la direction de déplacement horizontal sont très faibles.

Pour les pas 1 (passage de 0 à $59 \mathrm{~N}$ ) et 3 (passage de 144 à $315 \mathrm{~N}$ ), on obtient des écarts sur les déplacements en accord avec la précision estimée du dispositif. En effet, ils ne dépassent pas $0,2 \mathrm{~mm}$ pour le déplacement vertical et $0,6 \mathrm{~mm}$ pour le déplacement horizontal. Au pas 2 (passage de 59 à 144 N), nous voyons que ces écarts sont supérieurs à ceux constatés aux pas 1 et 3 .

Pour le chargement total, il est néanmoins possible de descendre en dessous d'un intervalle identifiable d'autocontrainte de 600 avec le déplacement vertical. De plus, malgré un chargement peu élevé de $171 \mathrm{~N}$, le pas 3 permet d'identifier un intervalle d'autocontrainte de 250 avec le déplacement vertical. C'est-à-dire avec une précision équivalente à la méthode utilisant la mesure des efforts dans notre cas expérimental.

Plutôt que de nous contenter des résultats obtenus avec les écarts moyens, nous décidons d'adopter une stratégie explorant la possibilité de cibler des nœuds particuliers. Pour le pas 3 qui donne globalement les meilleurs résultats, nous regardons la précision qui peut être atteinte sur les nœuds dont l'étude numérique nous a montré qu'ils étaient les plus sensibles à la variation d'autocontrainte. Ils correspondent aux nœuds qui ont les déplacements maximums en fonction du chargement : il s'agit des nœuds 10 et 14 pour le déplacement vertical et du nœud 15 pour le déplacement horizontal. La figure 8 nous permet d'écrire les équations suivantes pour une variation de $a$ égale à 1000 :

$$
\begin{aligned}
& D_{V-1000}\left(10^{-4} m\right)=0,058 \times C_{6}(N) \\
& D_{V-1000}\left(10^{-4} m\right)=0,054 \times C_{6}(N)
\end{aligned}
$$

Le rapport entre les différences de déplacements $D$ et les écarts constatés entre les champs de déplacements mesurés et simulés pour les nœuds considérés nous permet de déterminer l'intervalle $\Delta a$ identifiable. Verticalement, l'écart pour le nœud 10 est de $0,235 \mathrm{~mm}$ et il est de $0,042 \mathrm{~mm}$ pour le nœud 14 : cela conduit à un $\Delta a$ identifiable de 240 pour le nœud 10 et de moins de 50 pour le nœud 14. Horizontalement, l'écart pour le nœud 15 est de $0,233 \mathrm{~mm}$ et donc le $\Delta$ a identifiable est de 250. En ciblant ces nœuds particuliers, nous parvenons ainsi à réduire l'erreur sur l'identification de l'état d'autocontrainte de manière significative par rapport à l'utilisation des différences moyennes des déplacements sur les 15 nœuds.

Nous avons donc vu que plusieurs stratégies s'offrent à nous pour identifier le niveau d'autocontrainte avec le champ de déplacements des nœuds sous chargement statique. L'utilisation du champ de déplacements total moyen ne donne de bons résultats que si et seulement si l'essai n'a été entaché d'aucun aléas expérimental remettant en cause la linéarité de l'évolution du champ en fonction du chargement. 
C'est pourquoi il est raisonnable d'effectuer plusieurs pas de chargement pour réaliser l'identification soit sur l'ensemble des pas et sur les nœuds les plus sensibles, soit sur l'ensemble des nœuds sur le pas le plus adéquat. On peut aussi privilégier une identification ponctuelle sur les nœuds qui ont l'avantage d'être ceux qui se déplacent le plus et qui sont les plus sensibles à la variation des niveaux d'autocontrainte. Nous avons ainsi à notre disposition tous les éléments pour effectuer une identification la plus précise possible.

\section{Conclusion}

Nous avons pu constater que la structure soumise à un chargement vertical subissait un mouvement d'ensemble comparable à une rotation, probablement due à la dissymétrie des conditions d'appuis et de l'autocontrainte appliquée.

Pour la mesure des champs de déplacement des nœuds sous chargement statique, nous avons pu obtenir une bonne précision dans la prédiction du champ de déplacements. Ces résultats sont suffisamment prometteurs pour utiliser directement ces mesures de champ pour identifier l'autocontrainte des grilles de tenségrité en s'affranchissant des mesures plus lourdes des efforts dans les différents éléments.

Cependant, la précision du positionnement de la cible sur chaque nœud ne permet pas de conclure quant à la pertinence du déplacement horizontal pour réaliser cette identification. Une amélioration de la précision sur le positionnement horizontal des cibles placées sur les nœuds est donc à envisager. Avec des chargements plus élevés, elle doit permettre d'atteindre des mesures de champs de déplacements sous chargement statique plus exploitables. On peut aussi envisager de laisser les cibles fixées sur des nœuds particuliers tout le long du chargement. L'identification du niveau d'autocontrainte de la minigrille peut alors se faire avec une précision comparable avec celle obtenue grâce à la mesure des efforts de ses éléments. De plus, une structure réelle avec une plus grande portée, devrait donner des champs de déplacements plus importants et donc moins sensibles à la précision de mesure.

Il reste cependant à régler la délicate question de l'identification du rapport $a / b$ à l'aide des ces mesures en trouvant un critère sensible à sa variation : on envisage par exemple de réaliser une étude sur l'influence de la répartition des nœuds chargés sur le champ de déplacements mesuré.

Après ces améliorations effectuées, le tachéomètre devrait permettre de contrôler l'état d'autocontrainte au cours du temps et de diagnostiquer d'éventuels défauts de tension. 


\section{Bibliographie}

Angellier N., «Etude de l'influence de la géométrie et de la déformée d'une grille de tenségrité sur l'identification de son état d'autocontrainte », 25èmes Rencontres de Génie Civil "Conception et vie des ouvrages», Bordeaux, 23-25 mai 2007, Articles sur CDRom.

Angellier N., Dubé J.F., Crosnier B., « Comparison between experimental tests and numerical calculations carried out on a tensegrity mini grid», International Symposium «New olympics new shell and spatial structures», Beijing (China), 16-19 octobre 2006, IASS ed(s), p. CDrom 10p. texte complet, p. 214-215 résumé.

Averseng J., Mise en œuvre et contrôle des systèmes de tenségrité, Thèse de doctorat, Université Montpellier 2, 2004.

Averseng J., Méthodologie de la mise en auto-contrainte des systèmes de tenségrité, DEA, Université Montpellier 2, 2001

Averseng J., Crosnier B., « Prestressing tensegrity systems - Application to multiple selfstress state structures », International Journal of Structural Stability and Dynamics, vol. 4, $\mathrm{n}^{\circ} 4$, 2004, p. 543-557.

Averseng J., Crosnier B., «Static and dynamic robust control of tensegrity systems », Journal of the International Association for Shell and Spatial Structures, vol. 45, $\mathrm{n}^{\circ} 3$, 2004, p. 169-174.

Motro R., « Tensarch Project», First International Conference on Space Structures, In : Telford T, editor. Guilford, UK : G.A.R. Park and P. Disney, 2002, p. 57-66.

Motro R., Tensegrity, Kogan Page Science, 2003.

Quirant J., Systèmes de tenségrité et autocontrainte : qualification, sensibilité et incidence sur le comportement, Thèse de doctorat, Université Montpellier 2, 2000.

Quirant J., Kazi Aoual M.N., Laporte R., « Tensegrity systems: the application of linear programmation in search of compatible selfstress states », Journal of the International Association for Shell and Spatial Structures, vol. 44, $\mathrm{n}^{\circ}$ 1, 2003, p. 33-50.

Sanchez. L. R., Contribution à l'étude mécanique des systèmes de tenségrité, Thèse de doctorat, Université Montpellier 2, 2005.

Verpeaux P., Charras T., Millard A., Castem2000, une approche moderne du calcul des structures. In J.M Fouet, P. Ladevèze, and R. Ohayon, editors, Calcul des structures et intelligence artificielle, 1998. 